\title{
Cytokinin signalling regulates two-stage inflorescence arrest in Arabidopsis
}

\author{
Catriona H. Walker ${ }^{1}$, Alexander Ware ${ }^{2}$, Jan Šimura ${ }^{3}$, Karin Ljung ${ }^{3}$ Zoe Wilson ${ }^{2}$, Tom \\ Bennett $^{1+}$
}

1. School of Biology, Faculty of Biological Sciences, University of Leeds, Leeds, LS2 9JT.

2. School of Biosciences, University of Nottingham, Loughborough, Leics, LE12 5RD.

3. Department of Forest Genetics and Plant Physiology, Umeå Plant Science Centre, Swedish

University of Agricultural Sciences, 90183 Umeå, Sweden

+ Address for correspondence: t.a.bennett@leeds.ac.uk

\section{ABSTRACT}

2 To maximise their reproductive success, flowering plants must correctly time their entry into and

3 exit from the reproductive phase (flowering). While much is known about the mechanisms that

4 regulate the initiation of flowering, the regulation of end-of-flowering remains largely

5 uncharacterised. End-of-flowering in Arabidopsis thaliana consists of the quasi-synchronous arrest

6 of individual inflorescences, but it is unclear how this arrest is correctly timed with respect to

7 environmental stimuli and ongoing reproductive success. Here we show that Arabidopsis

8 inflorescence arrest is a complex developmental phenomenon which includes a decline in size and

9 cessation of activity in the inflorescence meristem (IM), coupled with a separable developmental

10 arrest in all unopened floral primordia (floral arrest); these events occur well before the visible

11 arrest of the inflorescence. We show that global removal of inflorescences can delay both IM arrest

12 and floral arrest, but that local fruit removal only delays floral arrest, emphasising the separability of

13 these processes. We test a role for cytokinin in regulating inflorescence arrest, and find that

14 cytokinin treatment can delay arrest. We further show that gain-of-function cytokinin receptor

15 hypersensitive mutants can delay floral arrest, and also IM arrest, depending on the expression

16 pattern of the receptor; conversely, loss-of-function mutants prevent extension of flowering in

17 response to inflorescence removal. Collectively, our data suggest that the dilution of cytokinin

18 among an increasing number of sink organs leads to end-of-flowering in Arabidopsis by triggering

19 IM and floral arrest, conversely meaning that a lack of reproductive success can homeostatically

20 extend flowering in compensation. 


\section{INTRODUCTION}

22 In order to maximise reproductive success, flowering plants must simultaneously fulfil three distinct requirements. Firstly, the quantity of reproductive structures produced by the plant inflorescences, flowers, fruits and seeds - must be carefully matched to the availability of resources (light, fixed carbon, nitrate, phosphate and water), both those already acquired by the plant, and those it might yet acquire [1]. Secondly, the timing of both the start and end of the reproductive phase must be optimised to occur in the correct season, and to coincide with the availability of both pollinators and crucially, potential mates. Thirdly, plants must measure their own reproductive success, and use this information to modify both the quantity of reproductive structures they produce, and the timing of their reproductive phase [1]. We can observe that all of these criteria are met, producing a coherent and flexible 'reproductive architecture' that can react to changes in circumstance [2], but our mechanistic understanding of reproductive architecture control is still limited.

However, given our knowledge of shoot branching control in flowering plants, it is very likely that the integration of long-distance hormonal signals plays a key role in determining the quantity of reproductive structures produced. For instance, soil nitrate and phosphate availability respectively upregulate cytokinin synthesis and downregulate strigolactone synthesis [3,4]. Cytokinin and strigolactones are transported root-to-shoot, and are perceived in axillary buds to determine their outgrowth, respectively promoting and repressing outgrowth [5], and thus connecting quantity of branches to soil resources. Apical dominance, which is driven by export of the hormone auxin from actively-growing shoot apices, also plays a key role in shoot branching regulation by inhibiting the activation of additional axillary buds through the self-organising properties of the auxin transport system $[6,7,8,9]$. Removing actively growing shoots removes this inhibition, and allows new axillary buds to activate and accurately replace the lost branches [2]; apical dominance thus acts as a mechanism by which plants can 'measure' their shoot branching. There is certainly evidence that both fruit and seeds can also act as sources of 'dominance' within the reproductive system [10], and can prevent new fruit, seed and inflorescences from developing [1,2], likely also through their export of auxin $[10,11,12,13,14]$. Furthermore, cytokinin has been shown to mediate the connection between soil nitrate resources and the activity of inflorescence meristems, which initiate new floral meristems at a greater rate ('florochron') as nitrate levels increase [15].

The timing of reproduction - or at least its initiation - is generally very well understood in flowering plants. At least seven distinct environmental or internal cues are integrated together to regulate the floral transition that begins the reproductive phase $[16,17]$. However, the events that contribute to end-of-flowering are generally much less studied, perhaps in part because end-of-flowering is a much more diverse phenomenon than floral transition [18]. While floral transition is a single 
occur, and different species use them in different combinations to end their reproductive phase

60 [18]. In Arabidopsis, end-of-flowering occurs because plants cease to initiate new inflorescences early in flowering, and because each inflorescence has a finite developmental lifetime [12]. End-offlowering in Arabidopsis was initially proposed to be a synchronised 'global proliferative arrest' [19], but recent work demonstrates that each inflorescence stops opening new flowers as locallymediated process ('inflorescence arrest') that can occur independently of other inflorescences [12]. The quasi-synchronous nature of inflorescence arrest in Arabidopsis is mostly explained by the quasi-synchronous initiation of inflorescences [12]. The timing of inflorescence arrest can be modified by both local and systemic feedback from fertile fruit and inflorescences, forming a flexible system in which developmental timing and measurement of reproductive success are coupled $[12,19,20]$.

Most studies have viewed inflorescence arrest as resulting from the arrest of the inflorescence meristem (IM) itself $[19,20,21]$. Certainly the IM does undergo a regulated arrest toward end-offlowering, before entering a quiescent 'dormancy-like' state [20] and then undergoing a gradual senescence [22]. It is also the case that extending the activity of the IM through genetic manipulations in key regulatory genes such as FRUITFULL can delay overall inflorescence arrest [21]. However, it is unclear whether the normal end of flower opening in inflorescences is directly caused by IM arrest. Certainly, the floral meristems (FMs) in Arabidopsis can also undergo their own arrest ('FM arrest'), and visible inflorescence arrest could be a result of this process, rather than directly due to IM arrest [18]. Hensel et al [19] showed that male sterility, and inflorescence/fruit removal (both before and after inflorescence arrest) could extend the lifetime of inflorescences, either by delaying inflorescence arrest, or undoing arrest if it had already occurred. However, it is unclear how the changes in inflorescence arrest in these treatments are actually mediated at a developmental level. In our previous work, we showed that auxin exported from

83 fertile fruits is required for timely inflorescence arrest [12], but again, did not identify which tissue is 84 responding to this signal. In the present study, we therefore aimed to define the developmental processes underlying inflorescence arrest in Arabidopsis, and to understand in particular the mechanisms by which local and systemic measurement of reproductive success is integrated into 87 these developmental processes. 


\section{Arabidopsis inflorescence arrest consists of separate inflorescence meristem} and floral arrest stages

To define how Arabidopsis inflorescences arrest, we grew a large population of wild-type Col-0 plants. Each plant was sampled at a given timepoint after visible floral transition ('bolting') and was destructively analysed to determine 1) the number of opened flowers; 2) the number of as-yetunopened floral primordia and buds; and 3) the total number of floral nodes (i.e. the sum of 1 and 2) on the primary inflorescence $(\mathrm{PI})$ at each timepoint. In this experiment, we observed that flower opening is a strongly linear process, with plants opening $\sim 3$ flowers/day from $6 \mathrm{dpb}$ (i.e. anthesis) until 17dpb (Figure 1B), at which point the inflorescence arrests. We found that the initiation of floral nodes proceeds at the same linear rate, indicating that flowers mature at a constant rate after initiation (Figure 1A). At the 0dpb timepoint, we found that inflorescences had already formed $\sim 18$ primordia, suggesting floral transition actually occurred 6 days before visible bolting. The initiation of floral nodes continued at 3/day, until it plateaued at $12 \mathrm{dpb}$ (Figure $1 \mathrm{~A}$ ). This demonstrates that the IM stops initiating new floral primordia 5 days before visible inflorescence arrest, and that in the final phase, the inflorescence is only opening existing floral buds, and not initiating new ones. Our data thus indicate that Col-0 inflorescence lifetime consists of two overlapping stages; an IM-driven flower initiation phase, and an FM-mediated flower-opening phase (Figure 1A,B)

Consistent with these data, the number of as-yet-unopened floral primordia initially increases until flowers (Figure 1C). The number of primordia then begins to decline from $12 \mathrm{dpb}$, since no new floral primordia are being initiated, but flowers continue to open. Primordia number then plateaus again at $17 \mathrm{dpb}$, after the opening of the final flowers, and the inflorescence arrests with cluster of 15 unopened buds/primordia (Figure 1C, E). Thus, the final set of primordia initiated from 8$12 \mathrm{dpb}$ do not open, and the timing of IM arrest does not determine the timing of inflorescence arrest.

Surprisingly, these data show that last flower to fully mature at $17 \mathrm{dpb}$ was therefore initiated at $7 \mathrm{dpb}$, just after anthesis and $\sim 5$ days before IM arrest (Figure 1). The maturation state of the oldest unopened flowers within the bud cluster, including the presence of sepals enclosing the bud (Figure 1E), shows that unopened primordia undergo arrest when these oldest of these primordia are $\sim 6$ days old [23]. Since these oldest unopened primordia initiated $\sim 4$ days before IM arrest, it follows that FM arrest must have occurred $\sim 2$ days after IM arrest itself (Figure 1). 
126 constant until approximately 6dpb (i.e. anthesis) and then showed two distinct stages of decline in

127 diameter, with the first occurring between 7-12dpb, until the point of IM arrest. After IM arrest, there

128 is a short plateau before a second decline between 15-17dpb, until the point of inflorescence

129 arrest. Thus, physical changes in the IM mirror the discrete stages of inflorescence arrest we have

130 identified. Our results are consistent with recent work which shows the same decline in IM size

131 over inflorescence lifetime [22], but provide a more refined time-sequence and more nuanced 132 results. 
A
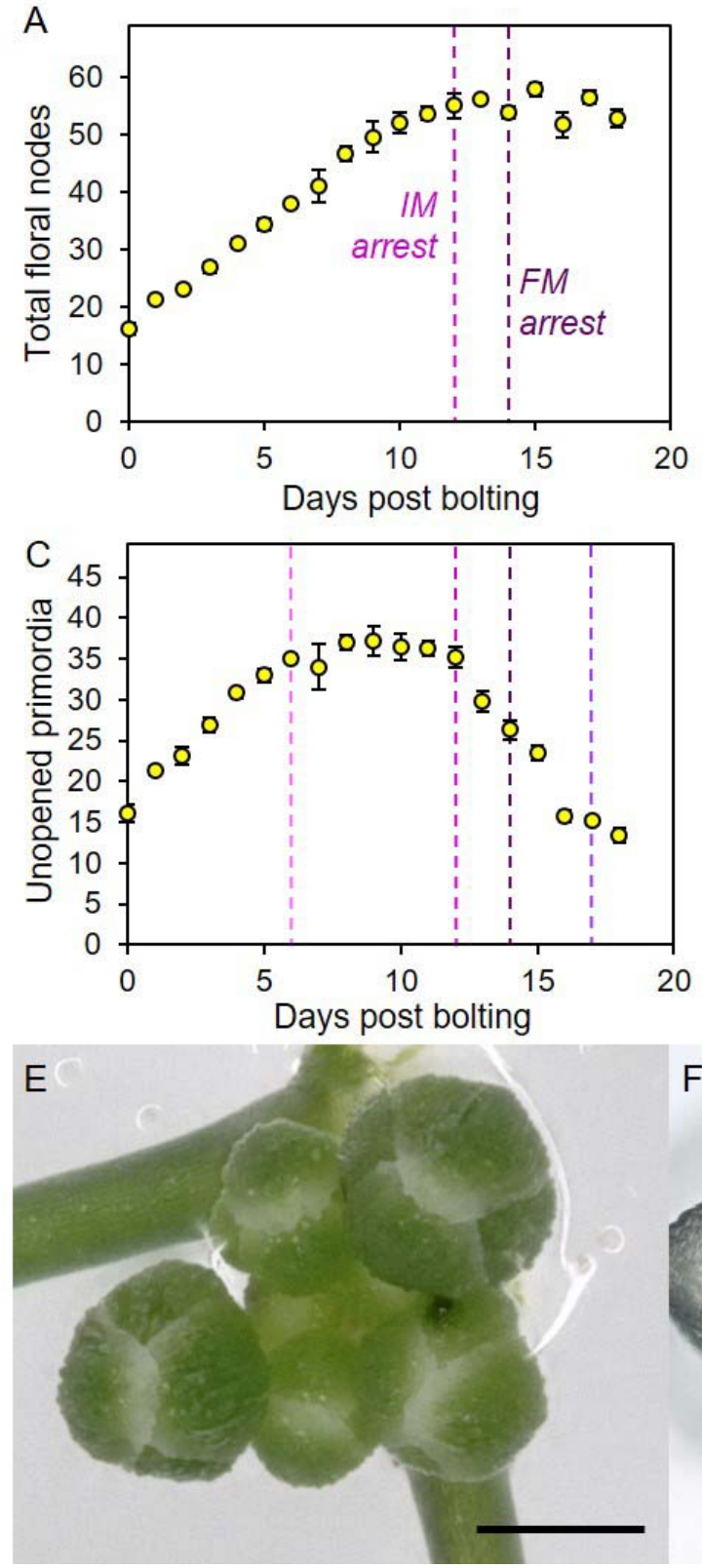
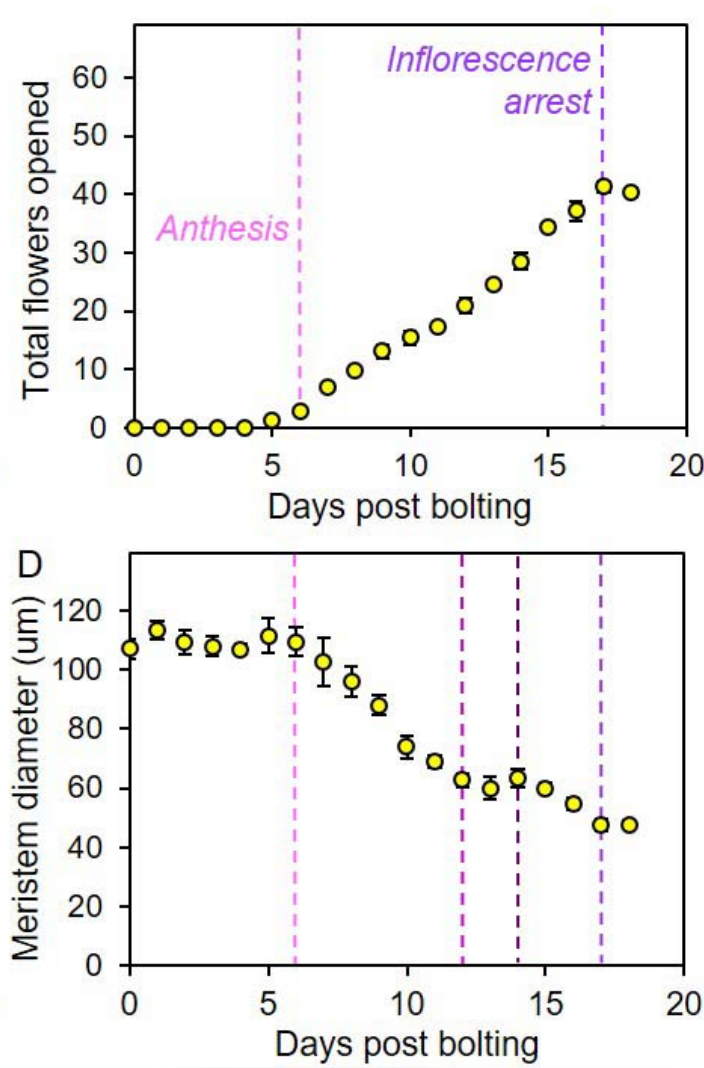

$\mathrm{F}$

Days post bolting

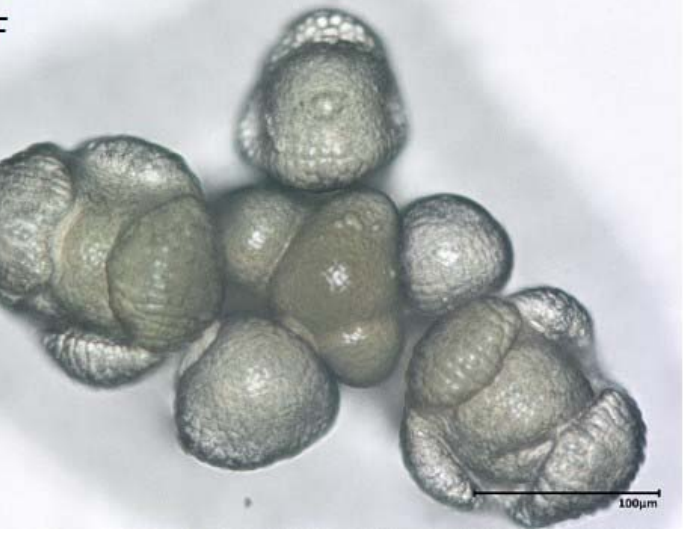

\section{Figure 1: Inflorescence arrest is a two-stage process}

134 Plants were grown in a controlled environment chamber, and assigned randomised collection

135 dates. Samples were collected daily from the primary inflorescence from 0 days post bolting (dpb) 136 onwards.

137 (A-C) Scatter graphs showing number of floral nodes (A), the number of opened flowers (including 138 previously opened flowers)(B) and the number of unopened floral buds and primordia (C) present 139 along the primary inflorescence on each day post bolting. (D) Scatter graph showing mean IM 140 diameter on each day post bolting. Error bars indicate s.e.m. The dashed vertical lines indicate the 141 key points in inflorescence lifetime highlighted by this analysis: anthesis (6 dpb), IM arrest (12 dpb)

142 and inflorescence arrest (17 dpb). (E) Image showing a typical example of floral buds present 143 within the bud cluster following the final flower opening. Scale bar $=500 \mu \mathrm{m}$. (F) Image showing IM 
144 and remaining attached floral primordia. The meristem is in the centre of the bud cluster, with

145 progressively older floral primordia spiralling outwards. Scale bar $=100 \mu \mathrm{m}$.

146 Global inflorescence removal prolongs IM activity; local fruit removal 147 prolongs flower opening

148 Our data demonstrate that inflorescence arrest is not a single developmental event, but do not 149 establish the regulatory relevance of these events. We hypothesised that floral arrest and IM arrest

150 are separable processes that are differentially regulated in response to environmental or internal

151 stimuli. To test this idea, we performed different treatments on Col-0 plants that, based on previous

152 reports, we hypothesised would increase floral duration of the primary inflorescence. Firstly, we

153 continuously removed all inflorescences except the PI from plants $6 \mathrm{dpb}$ onwards, prior to IM arrest

154 [19], and secondly, we continuously removed fruit from the PI alone from 14dpb, prior to floral

155 arrest [12].

156

157 We initially examined the rate of flower opening ('florochron') on the PI of Col-0 plants, which 158 showed that both these treatments indeed increased the floral duration of the PI compared to 159 untreated plants, which in this experiment underwent inflorescence arrest at $\sim 24 \mathrm{dpb}$ (Figure 2A).

160 Removing inflorescences from $6 \mathrm{dpb}$ resulted in an additional $\sim 25$ flowers opening, due to 161 prolonged duration (by $\sim 8$ days), rather than increased rate of opening (Figure $2 \mathrm{~A}$ ). Removing fruit 162 from $14 \mathrm{dpb}$ also resulted in prolonged duration ( $\sim 10$ days), but with a slower rate of flower opening 163 ( 15 additional flowers at $\sim 1.5 /$ day) (Figure $2 \mathrm{~A})$.

The qualitative differences between these treatments suggested that their effects arose from different developmental events. We therefore examined the timing of IM and inflorescence arrest in plants subjected to these treatments, using the same basic experimental design as in Figure 1. In this experiment, untreated plants underwent IM arrest at 18dpb (Figure 2C-F), and inflorescence arrest at $\sim 22 \mathrm{dpb}$ (Figure $2 \mathrm{C}$ ). Plants treated with inflorescence removal from $6 \mathrm{dpb}$ showed a clear delay in IM arrest, continuing to initiate floral nodes for 5-6 days after control plants, and ultimately producing significantly more floral nodes (e.g. day 26: t-test, $p<0.05, n=5$ )(Figure $2 C, D$ ). Consistent

172 with this, plants also showed a delay in reduction of IM size between 8-16dpb (Figure 2B).

173 Intriguingly, these plants also showed a clear delay in inflorescence arrest, even accounting for the

174 delay in IM arrest; the plants continued to open new flowers for 10 days after IM arrest (until $175 \sim 32 \mathrm{dpb}$ ), and arrested with a bud cluster of only 5 primordia. Thus, compared to control plants, the 176 treated plants flowered for 10 days longer, initiated an additional 15 flowers, and opened an 177 additional 25 (Figure 2A-F).

179 In contrast, plants treated with local fruit removal after 14dpb showed no clear alteration in the 180 timing of IM arrest (Figure 2C,D), but did have a small and statistically non-significant increase in 181 floral node number (by $\sim 4$ nodes)(e.g. day 26: t-test, $p>0.05, n=5$ )(Figure 2C,D). Conversely, they 
182 showed a very clear delay in inflorescence arrest, continuing to open flowers for an additional $\sim 14$

183 days until $36 \mathrm{dpb}$, resulting in the opening of an additional $\sim 15$ flowers, at which point the bud

184 cluster was essentially exhausted (Figure 2E,F). Thus, while early global inflorescence removal

185 delayed both IM and FM arrest, local fruit removal only delayed FM, with no obvious change in IM

186 activity (Figure 2D). These data therefore show that the two stages of inflorescence arrest are

187 separable and functionally distinct; dramatic, global loss of inflorescences/fruit can prolong IM and

188 FM activity, but local fruit removal prolongs FM activity. 

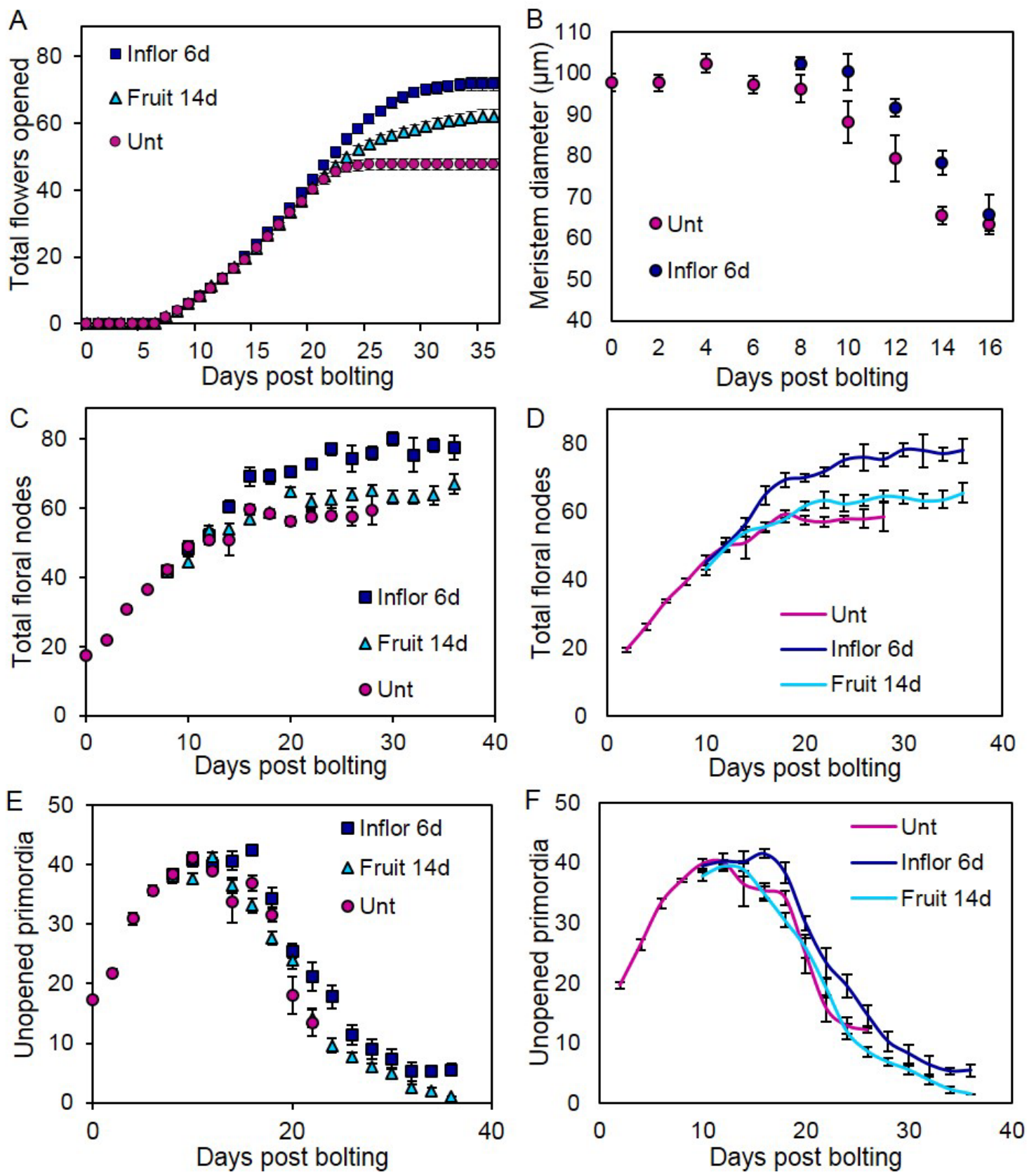

189 Figure 2: Systemic and local stimuli increase and extend flower opening

190 Based on observations in Figure 1, we performed 4 different treatments on flowering Col-0 plants.

191 Firstly, we removed all inflorescences apart from the primary inflorescence (PI) from the plant at 6

192 days post bolting (Inflor 6d). This timepoint was chosen as the earliest timepoint at which

193 secondary inflorescences are visibly elongating. We also removed all fruits from the PI at 14 days

194 post bolting and continuously thereafter (Fruit 14d). This timepoint was chosen as the earliest

195 timepoint at which sufficient numbers of developed fruit are present in order for their removal to

196 potentially make a difference.

197 (A) Scatter graph of cumulative flowers opened on the PI of each treatment each day post bolting;

198 data collected non-destructively from individual plants. Error bars show s.e.m., n=10-13. 
199 (B-F) A large population of Col-0 plants were grown under controlled conditions. The timing of 200 visible bolting was recorded for each plant. Plants were randomly assigned to be sampled on a 201 given number of days post-bolting, and then destructively sampled at that timepoint. Timepoints 202 were spaced every two days, and 3-12 plants sampled for each timepoint. Error bars for all graphs 203 show s.e.m. (B) Scatter graph showing mean IM diameter, from 0-16dpb for control plants, and 204 day 6-16 from plants treated from day 6 with inflorescence removal. (C) Scatter graph showing the 205 number of total floral nodes present from $0-28 \mathrm{dpb}$ for control plants, and from day 6-36/38 dpb for 206 plants treated from day 6 with inflorescence removal or day 14 with fruit removal. (D) Scatter graph 207 showing the data from (C) plotted as a two-timepoint rolling average in order to show a slightly 208 smoothed version of the data illustrating the overall trend. (E) Scatter graph showing the total 209 number of unopened floral primordia present in the inflorescence apex from 0-30dpb for control 210 plants, and from day 6-36/38 dpb for plants treated from day 6 with inflorescence removal or day 21114 with fruit removal. Buds and primordia were counted by dissecting buds from the bud cluster 212 under a dissecting microscope. (F) Scatter graph showing the data from (E) plotted as a two213 timepoint rolling average in order to show a slightly smoothed version of the data illustrating the 214 overall trend. 


\section{Global branch removal and local fruit can reactivate IM and FM activity}

216 Hensel et al [19] observed that Arabidopsis inflorescences sometimes naturally reactivate, which

217 we found to occur in $\sim 50 \%$ of Col-0 plants, typically those producing slightly fewer fruits during

218 initial flowering (Figure S1A). Hensel et al [19] also showed that individual inflorescences can also

219 be induced to reactivate in response to post-arrest inflorescence or fruit removal. Given our new

220 data, we questioned whether this occurs by reactivation of IM activity, FM activity, or both. We

221 therefore treated Col-0 plants with global inflorescence removal after arrest of the $\mathrm{PI}$, which

222 promoted re-activation after an $\sim 8$ day delay, beginning with $\sim 4$ of the characteristic 'failed' flowers

223 previously reported [19](Figure 3B), before successful opening of $\sim 9$ new fertile flowers (Figure

$2243 \mathrm{~A}$ ). Treated plants did not produce any additional floral nodes in total (Figure 3A)(t-test, $p>0.05$,

$225 \mathrm{n}=6-10)$, showing these changes are achieved by re-activation of FM activity without new IM

226 activity. We also found that local fruit removal after arrest in Col-0 was able to trigger the same

227 level of re-activation of FM activity, although the process occured more quickly (within $~ 4$ days)

228 and with fewer failed flowers (Figure S1B). Again, this occurs without any increase in the number

229 of floral nodes initiated between treated and untreated plants (Figure S1C).

231 We also tested global inflorescence removal after arrest of the PI in the Ler ecotype, in which

232 Hensel et al performed their experiments. In contrast to Col-0, we found reactivation of both IM and

233 FM activity, with treated plants opening of an additional $\sim 33$ new fertile flowers (and 9 failed

234 flowers), but also showing an clear increase of $\sim 17$ total floral nodes over untreated plants (t-test,

$235 \mathrm{p}<0.0001, \mathrm{n}=9-11$ )(Figure S1D). This unexpected ecotypic difference in IM reactivation potential

236 between Col-0 and Ler is intriguing, and might reflect the known roles of ERECTA in meristem

237 maintenance $[24,25,26]$; it is possible that it is the erecta mutation itself that contributes to the

238 difference between the ecotypes. Irrespectively, these data again emphasise the separability of IM

239 and FM arrest as developmental processes. 


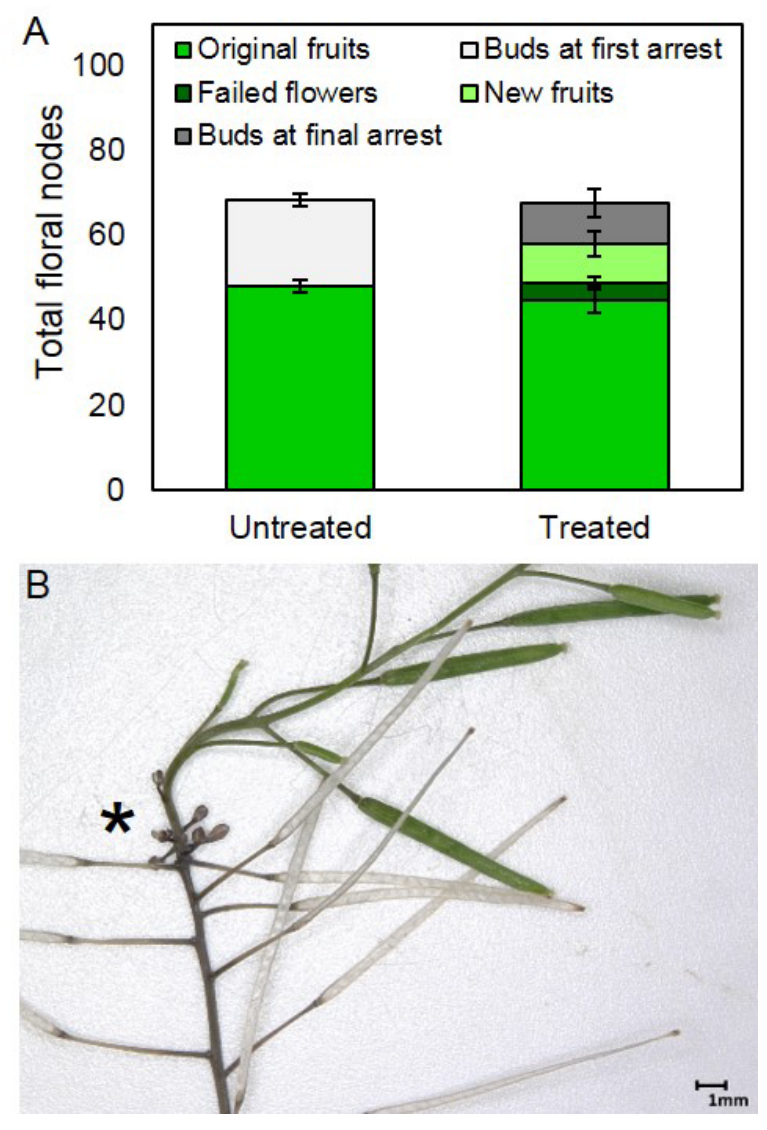

\section{Figure 3: Reactivation of FM activity by inflorescence removal}

241 A) Stacked bar graph, showing the number of floral nodes on the PI produced in Col-0 plants either 242 left untreated, or treated by removal of all other inflorescences after arrest of the PI. The total floral 243 nodes (i.e. the height of the full stack) is broken down into fruit produced on the PI during initial 244 flowering (mid-green, lower bars), plus either a) the number of buds and primordia remaining in the 245 bud cluster at first arrest (light grey)(untreated plants only), or b) the number of failed flowers (dark 246 green), new fertile fruits (light green) and the number of buds and primordia remaining in the bud 247 cluster at final arrest (dark grey)(treated plants only). Error bars indicate s.e.m, $n=10$ (untreated), 2486 (treated).

249 B) Photo showing within-inflorescence reflowering in Col-0, with older fruit dehisced, a small 250 cluster of characteristic failed flowers (asterisk) and then resumption of fertile flower opening. 

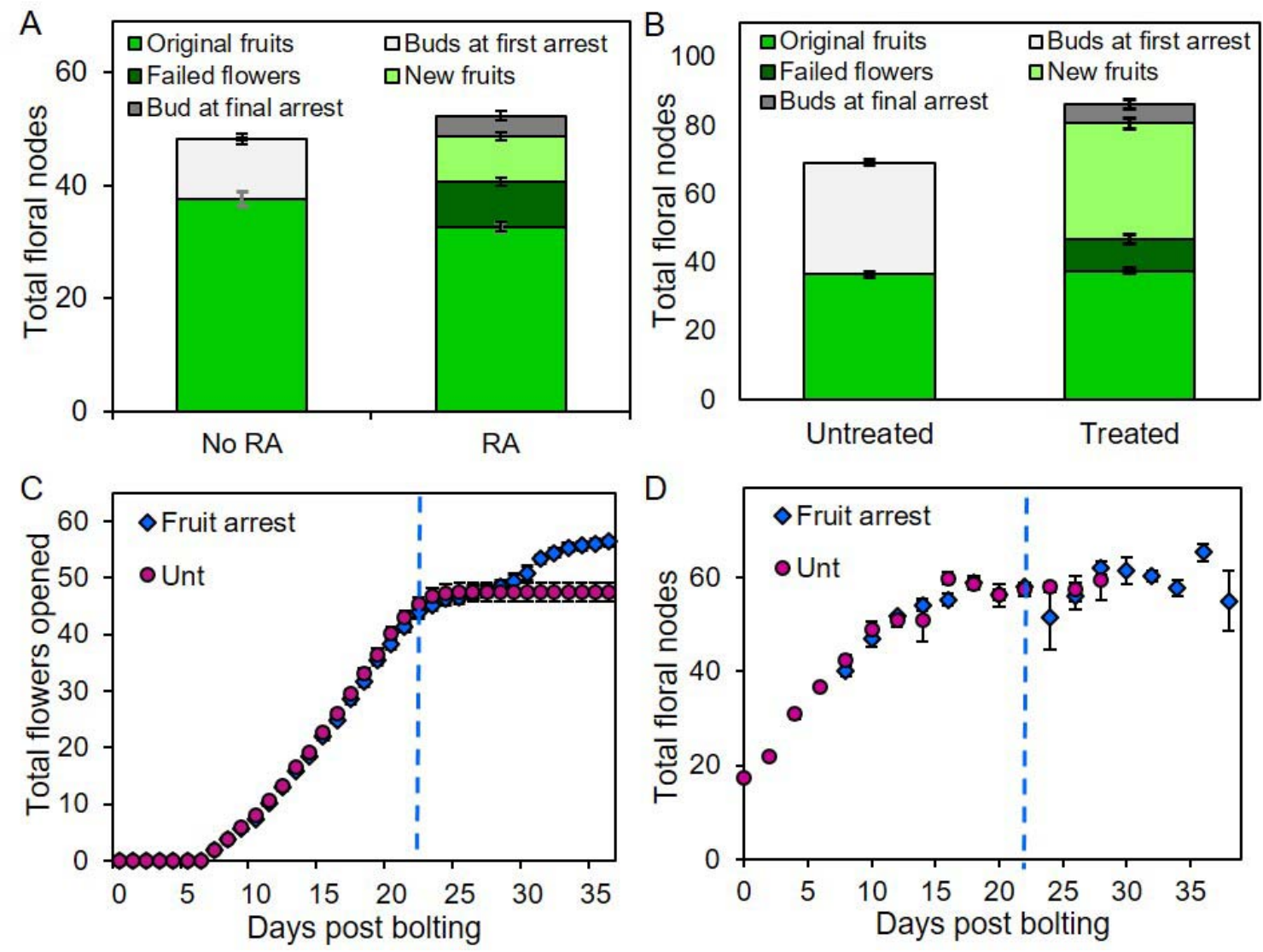

\section{Figure S1: Reactivation of IM and FM activity in Col-0 and Ler}

252 A) Stacked bar graph, showing the number of floral nodes on the PI produced in Col-0 plants left 253 untreated for sufficient time, which either reactivated (RA) or did not (No RA). The total floral nodes 254 (i.e. the height of the full stack) is broken down into fruit produced on the PI during initial flowering 255 (mid-green, lower bars), plus either a) the number of buds and primordia remaining in the bud 256 cluster at first arrest (light grey)(untreated plants only), or b) the number of failed flowers (dark 257 green), new fertile fruits (light green) and the number of buds and primordia remaining in the bud 258 cluster at final arrest (dark grey)(treated plants only). Error bars indicate s.e.m, $n=21$ (no RA), 20 259 (RA).

260 B) Stacked bar graph, showing the number of floral nodes on the PI produced in Ler plants either 261 left untreated, or treated by removal of all other inflorescences after arrest of the PI. The total floral 262 nodes (i.e. the height of the full stack) is broken down into fruit produced on the PI during initial 263 flowering (mid-green, lower bars), plus either a) the number of buds and primordia remaining in the 264 bud cluster at first arrest (light grey)(untreated plants only), or b) the number of failed flowers (dark 265 green), new fertile fruits (light green) and the number of buds and primordia remaining in the bud 266 cluster at final arrest (dark grey)(treated plants only). Error bars indicate s.e.m, 11 (untreated), 9 267 (treated). 
268 C) Scatter graph of cumulative flowers opened on the PI of each treatment. Data collected non-

269 destructively from 11 individual plants per treatment, assessed daily post bolting. 'Fruit arrest'

270 plants were treated from 1 day after their arrest by the removal of all fruit on the primary

271 inflorescence, then left to respond; 'Unt' plants were left untreated. The point of treatment for fruit

272 arrest plants has been normalised to 24 days post anthesis (grey dashed line), such that day 25

273 shows plants 1 day post-treatment, etc. Error bars show s.e.m.

274 D) Scatter graph of total floral nodes present on the PI of each treatment. A large population of

275 Col-0 plants were grown under controlled conditions; 'Fruit arrest' plants were treated from 1 day

276 after PI arrest by the removal of all fruit on the PI, then left to respond. 'Unt' plants were left

277 untreated. The timing of visible bolting was recorded for each plant; plants were then randomly

278 assigned to be sampled on a given number of days post-bolting (or post-arrest), and then

279 destructively sampled at that timepoint. Timepoints were spaced every two days, and 5-7 plants

280 sampled for each timepoint. Error bars for all graphs show standard error of the mean. The point of

281 treatment for fruit arrest plants has been normalised to 24 days post bolting (blue dashed line),

282 such that day 25 shows plants 1 day post-treatment, etc. Error bars show s.e.m. 


\section{Cytokinin signalling regulates IM arrest}

284 We previously showed that auxin export from 'late' fruit is required for inflorescence arrest [12];

285 given the data presented here, we are therefore confident that this auxin export is a key regulator

286 of FM arrest. However, IM arrest occurs too early to be caused by late fruit, and we have

287 previously shown that 'early' fruit have no impact on inflorescence arrest [12]. It therefore appears

288 unlikely that auxin dynamics regulate IM arrest. Cytokinin is an important root-shoot signal, the

289 availability of which has previously been shown to regulate IM activation and activity in relation to

290 environmental stimuli $[15,27]$. We therefore reasoned that IM arrest might be regulated by cytokinin

291 dynamics in the shoot system.

292

293 To test this idea, we firstly examined cytokinin signalling dynamics in the IM by confocal 294 microscopy, using the TCSn:GFP reporter line [28] to visualise the magnitude of cytokinin 295 signalling over the course of IM lifetime. In untreated plants, we saw a marked decrease in 296 cytokinin signalling in the IM between $3 \mathrm{dpb}$ and $15 \mathrm{dpb}$, the time-frame in which the IM typically 297 arrests (Figure 4A-H). Consistent with this, using qPCR we also observed concomitant reductions 298 in the expression of ARR5 and ARR7 in inflorescence apices over the same time frame (Figure 4I, 299 Figure S2A).

300

We next tested whether cytokinin treatment is sufficient to delay inflorescence arrest. We applied

$3021 \mathrm{mg} / \mathrm{g}$ cytokinin in lanolin to specific siliques at 12 days post anthesis. We observed a clear delay 303 of inflorescence arrest, with treated plants continuing to produce and open flowers long after 304 control plants had ceased to do so (Figure 4J). Application of $0.1 \mathrm{mg} / \mathrm{g}$ CK however had no obvious 305 effect, with inflorescence arrest and fruit number being the same as untreated plants, showing the 306 effect is strongly dose-dependent on cytokinin concentration (Figure S2B). Cytokinin at sufficiently 307 high levels is therefore able to extend flowering duration.

We next tested whether mutants with altered cytokinin signalling showed altered inflorescence arrest. We were particularly interested in the rock2 and rock3 mutants, which have increased cytokinin sensitivity, and have previously been described as producing more fruit along the main inflorescence before arrest; however, it was not entirely clear whether this was due to increased rate of development, or delayed arrest [29]. In addition to rock2 and rock3, we also examined the

314 arr1-4 mutant which has reduced cytokinin signalling in the shoot and the ipt3 ipt5 ipt7 (ipt357) 315 triple mutant which synthesises less cytokinin [27,30]. We observed that arr1-4 arrests $\sim 2$ days 316 earlier than Col-0, while ipt357 arrests at the same time, but having developed more slowly and 317 opened fewer flowers (Figure S2B). Conversely, we observed that rock2 arrested $\sim 5$ days later 318 than the WT under our conditions, while rock3 arrests an additional 5 days later that rock2 (Figure 319 S2C). Taken together, our results therefore strongly suggest that cytokinin regulates the duration of 320 inflorescence activity. 

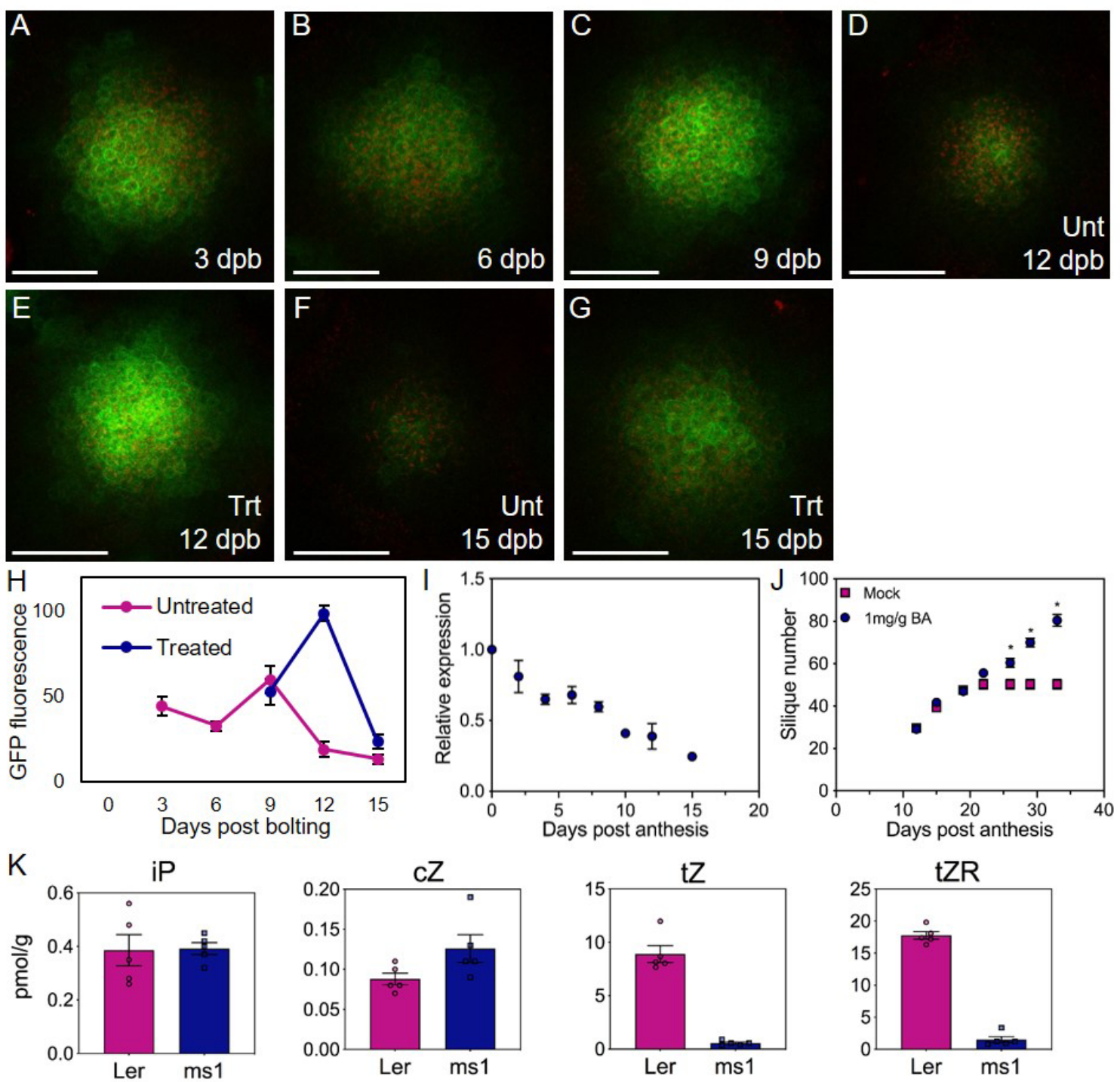

\section{Figure 4: Cytokinin signalling regulates IM arrest}

322 (A-G) Confocal microscopy images of primary IMs in Arabidopsis TCSn:GFP plants. GFP

323 fluorescence is shown in green, chloroplast autofluorescence in red. Images taken from IMs 324 dissected at $3(\mathbf{A}), 6(\mathbf{B}), 9(\mathbf{C}), 12(\mathbf{D}, \mathbf{E}), 15(\mathbf{F}, \mathbf{G})$ days post bolting (dpb). Plants were either 325 untreated $(\mathbf{A}-\mathbf{D}, \mathbf{F})$ or treated with removal of all secondary inflorescences at $6 \mathrm{dpb}(\mathbf{E}, \mathbf{G})$.Scale bars $326=50 \mu \mathrm{m}$.

327 (H) Quantification of relative GFP fluorescence (in arbitrary units) in primary IMs of Arabidopsis 328 TCSn:GFP plants between 3 and 15dpb, in untreated plants, or plants treated with removal of all 329 secondary inflorescences at $6 \mathrm{dpb}$. Data are means of $n=5-6$ meristems (except $9 \mathrm{dpb}$ treated, $n=2$ ), 330 error bars show s.e.m.

331 (I) Relative expression of ARR5 in inflorescence apices at different days post anthesis.

332 Quantification of the relative abundance of the transcript of ARR5 in inflorescence apices (all 333 unopened buds) in wild-type Col-0 plants harvested following the anthesis of the first flower (day 0 ) 
334 until inflorescence arrest (day 15) by qRT-PCR. Data are means of 4 biological replicates, error 335 bars show s.e.m.

336 J) Effect of cytokinin application to fruits on the primary inflorescence (PI) on the duration of 337 flowering, as measured by rate of fruit production. 12 days post anthesis with 6-benzlyaminopurine 338 (BA) dissolved in lanolin treatment at $1 \mathrm{mg} / \mathrm{g}$, or a mock treatment of lanolin only. Significant 339 differences between treatments at the same timepoint are indicated by asterisks (Sidak's multiple 340 comparisons, on a mixed-effects model, $\mathrm{p}<0.05, \mathrm{n}=8$ (mock), 7 (treated)).

341 (K). Concentration ( $\mathrm{pmol} / \mathrm{g}$ fresh weight) of the free cytokinin bases isopentenyladenine (iP), cis342 Zeatin (cZ), trans-Zeatin (tZ) (biologically active cytokinins) and trans-Zeatin riboside (tZR) (major 343 root-to-shoot transport form) in the fertile or sterile fruit of Ler and ms1 plant. $\mathrm{n} \square=\square 5$ biologically 344 independent samples (shown by overlying circles), error bars show s.e.m. 

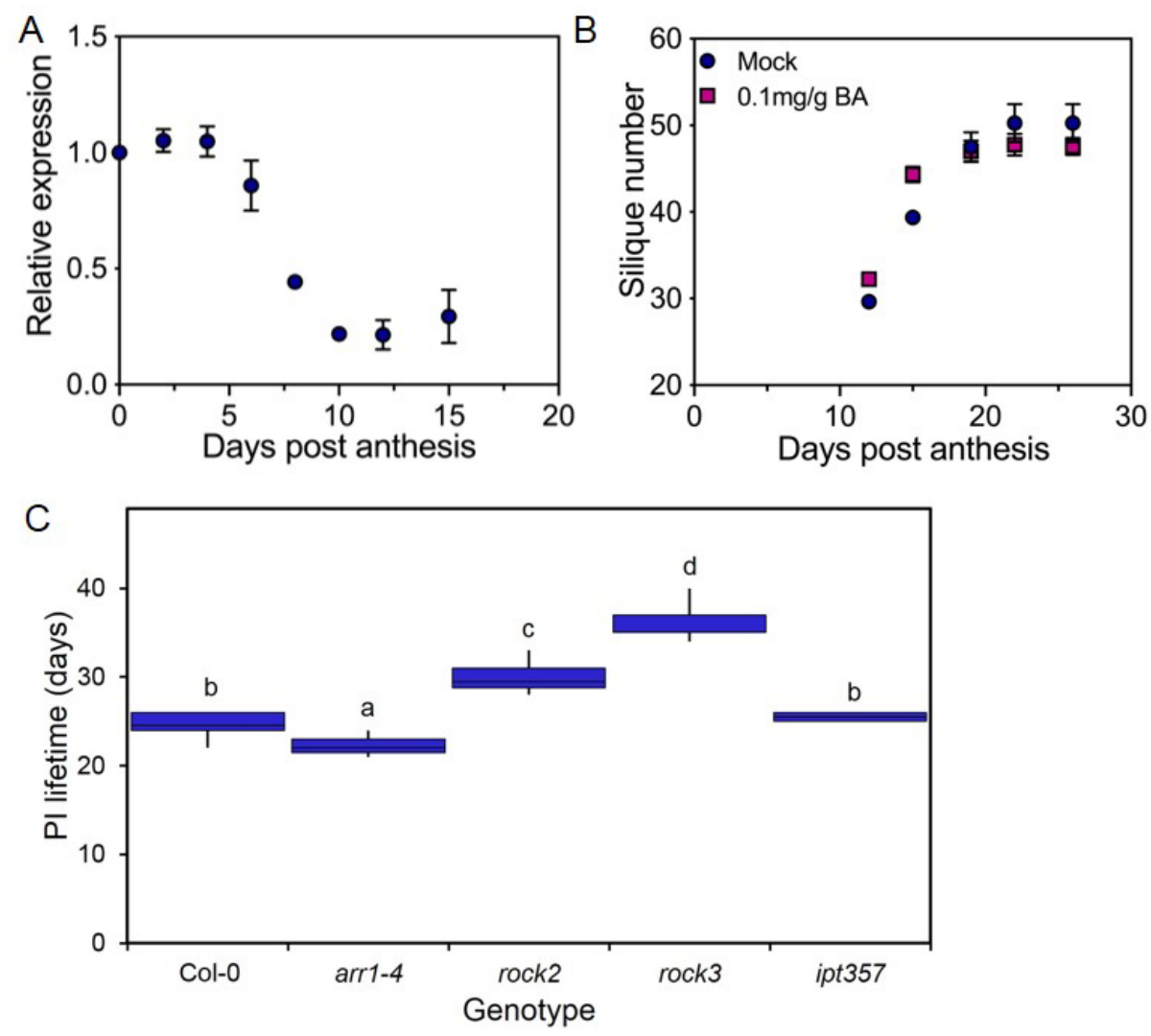

345 Figure S2: Cytokinin signalling regulates IM arrest

346 (A) Relative expression of ARR7 in inflorescence apices at different days post anthesis.

347 Quantification of the relative abundance of the transcript of ARR7 in inflorescence apices (all 348 unopened buds) in wild-type Col-0 plants harvested following the anthesis of the first flower (day 0 ) 349 until close to the end of flowering (day 15) by qRT-PCR.

350 (B) Effect of cytokinin application to fruits on the primary inflorescence (PI) on the duration of 351 flowering, as measured by rate of fruit production post anthesis. Fertile Ler plants were treated 352 from 12 days post anthesis with 6-benzlyaminopurine (BA) dissolved in lanolin treatment at $3530.1 \mathrm{mg} / \mathrm{g}$, or a mock treatment of lanolin only. No significant differences were observed between 354 treatments (Sidak's multiple comparisons, on a mixed-effects model, $\mathrm{p}<0.05, \mathrm{n}=8$ (mock), 9 355 (treated)).

356 (C) Box plot showing primary inflorescence lifetime (days) of Arabidopsis cytokinin mutants. Bars 357 with the same letter are not significantly different from each other (ANOVA, Tukey HSD test, $\mathrm{n}=4$ 358 12). Box indicates the interquartile range, internal line shows the median. Whiskers indicate 359 maximum and minimum values. 


\section{Cytokinin signalling mediates IM and FM arrest in response to local and} global developmental events

362 Based on our observations, we hypothesised that inflorescences and fertile fruit act as cytokinin sinks, and that the continued production of these new cytokinin sinks during flowering leads to a gradual reduction in free cytokinin levels, thereby triggering IM and FM arrest. In particular, we hypothesised that inflorescences and fertile fruit are sinks for trans-Zeatin ( $t Z$ ) cytokinin, which acts as a key root-to-shoot signal coupling root and shoot development [5]. Consistent with this idea, we found that wild-type fertile fruit have much higher levels of trans-Zeatin riboside ( $t Z R$ ) (the main transport form of $t Z$ )[31]), and the signalling-active $t Z$ form itself, compared to sterile fruit of the male sterile1 mutant [12](Figure 4K). Conversely, sterile and fertile fruit contained similar quanities of isopentenyladenine (iP) and cis-Zeatin (cZ) cytokinins, showing there is not a general reduction in cytokinin in sterile fruit (Figure 4K).

We therefore hypothesised that the dynamic response of IM and FM activity to global inflorescence and local fruit removal is explained by the reduction in the number of $t Z$ sinks. In the case of inflorescence removal, sufficient $t Z$ is liberated to prolong both IM and FM activity; in the case of local fruit removal, sufficient $t Z$ is liberated to prolong FM activity. Consistent with this idea, we found that plants treated with inflorescence removal at $6 \mathrm{dpb}$ showed a dramatic increase in cytokinin signalling at $12 \mathrm{dpb}$, before returning to pre-treatment levels (Figure $4 \mathrm{~A}-\mathrm{H}$ ); this strongly correlates with the observed extension in IM activity in plants subjected to this treatment (Figure 2).

To test this idea formally, we carefully examined the arrest phenotype, and response to global inflorescence removal and local fruit removal in rock2, rock3 and ahk2 ahk3 mutants. The mutants rock2 and rock3 have gain-of-function mutations in the cytokinin receptors ARABIDOPSIS HISTIDINE KINASE2 (AHK2) and AHK3 respectively, which confer increased cytokinin sensitivity [29]; the ahk2 ahk3 double mutant has a loss of function in both receptors, resulting in reduced cytokinin sensitivity $[32,33]$.

As in our earlier experiments, we tracked the number of floral nodes initiated, the number of opened flowers and the number of unopened buds and primordia on the PI for each genotype, over the course of inflorescence lifetime. Control Col-0 plants in this experiment behaved as expected from previous experiments. Plants underwent anthesis at $\sim 7 \mathrm{dpb}$ (Figure $4 \mathrm{~A}$ ), IM arrest at $\sim 15 \mathrm{dbp}$ (Figure 5B,C) and inflorescence arrest at $\sim 24 \mathrm{dpb}$ (Figure 5A,C). IM diameter decreased between anthesis and IM arrest, as also previously observed (Figure 5D). 
nodes initiated each day, leading to fewer flowers opening per day (2.3 vs 1.8 per day in Col-0 and ahk2 ahk3 respectively), and ultimately less nodes and flowers being formed (day 24: ANOVA + Dunnett's, $p<0.05, n=4)$. This is highly consistent with previous data showing that cytokinin controls the activity of the IM in response to environmental conditions [15].

\section{The Pls of rock3 behaved very similarly to Col-0 until inflorescence arrest, although they likely} underwent slightly earlier IM arrest than Col-0 (Figure 5B,C) producing less floral nodes in total (day 24: ANOVA + Dunnett's, $p<0.05, n=4-5$ ). However, rock3 plants continued opening flowers for longer than Col-0, until the bud cluster was almost extinct (Figure $5 \mathrm{C}$ ), opening $\sim 10$ more flowers in total (Figure 5A). The phenotype of rock3 is therefore qualitatively very similar to the effect of local fruit removal (Figure 2); there is no increase in IM activity, but a clear increase in FM activity.

The Pls of rock2 also behaved very similarly to Col-0 for the first 10 days of the experiment (Figure $5 A, B, C)$, at which point the rate of IM activity seemed to slow down slightly compared to Col- 0 , in (Figure 5B). However, they continued to initiate new floral nodes for longer than Col-0, with IM arrest delayed until $\sim 22 \mathrm{dpb}$ (Figure 5B,C), and eventually produced significantly more floral nodes that Col-0 (day 24: ANOVA + Dunnett's, $\mathrm{p}<0.05$, $\mathrm{n}=3-5$ )(Figure 5B). Furthermore, rock2 mutants also continued opening flowers for longer than Col-0, even taking into account the delay in IM arrest (Figure 5A,C). They open flowers for $\sim 14$ days after IM arrest, compared to $\sim 9$ days in Col-0, until the bud cluster was almost extinct. Overall, the phenotype of rock2 mutants is qualitatively similar to the effect of inflorescence removal in Col-0 plants; there is a delay in IM arrest, with more floral nodes initiated in total, and a subsequent additive delay in FM arrest, with a greater proportion of flowers ultimately opened.

The phenotype of rock 2 and rock3 indicate that cytokinin not only controls the rate of activity in the $\mathrm{IM}$, but also the timing of both IM and FM arrest in inflorescences. The phenotypes of rock2 and rock 3 are highly consistent with the expression patterns of $A H K 2$ and $A H K 3$. AHK2 is strongly expressed in both IMs and FMs, and rock2 affects the arrest of both IMs and FMs; $A H K 3$ is primarily expressed in FMs, and rock3 primarily affects the arrest of FMs [34]. Overall, our data support the hypothesis that cytokinin liberated by inflorescence or fruit removal causes changes in arrest, because plants that have increased cytokinin sensitivity show the same changes. We therefore hypothesised that, if this model is correct, then ahk2 ahk3 should fail to respond to either inflorescence or fruit removal, and that conversely rock2 and rock3 should over-respond to inflorescence removal -- but not to fruit removal, since they already open almost all flowers they produce.

To test these hypotheses, we performed inflorescence removal at $6 \mathrm{dpb}$ and fruit removal at $14 \mathrm{dpb}$ (consistent with Figure 2) treatments in ahk2 ahk3, rock2 and rock3 mutants. Consistent with our hypothesis, we found that ahk2 ahk3 showed very little response to either treatment, and no 
436 statistically significant difference in either the number of flowers opened or the overall lifetime of

437 the PI (Figure 5E,F). Similarly, we saw no significant difference in flowers opened or PI lifetime in 438 rock2 and rock3 in response to fruit removal (Figure 5E,F). However, we saw an increase the 439 number of flowers opened in both rock2 and rock3 compared to Col-0, thus strongly supporting our 440 hypothesis (Figure 5E). 

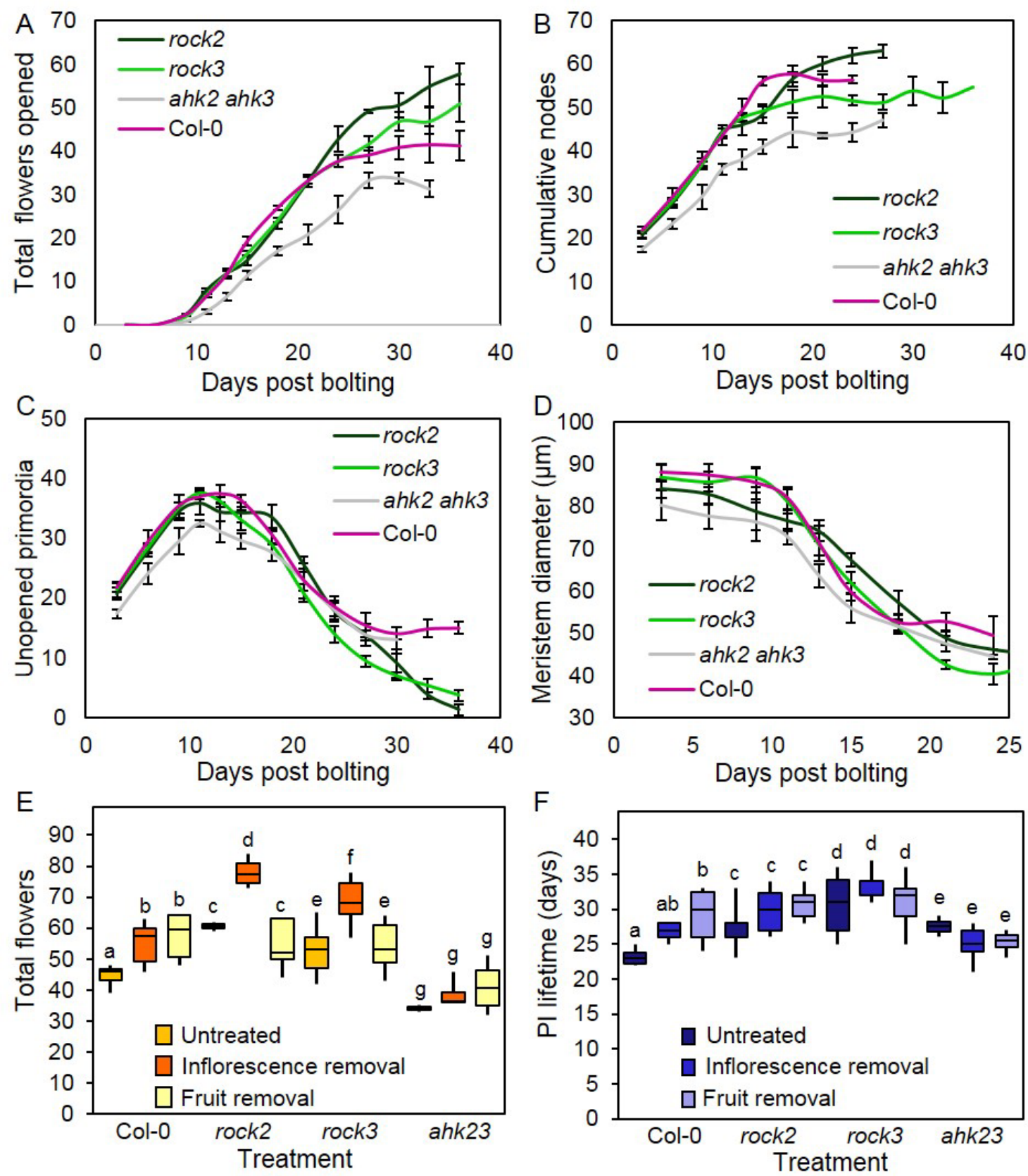


\section{Figure 5: Cytokinin signalling regulates floral arrest}

442 (A-D) Large populations of Col-0, ahk2 ahk3, rock2 and rock3 plants were grown under controlled 443 conditions. The timing of visible bolting was recorded for each plant. Plants were randomly 444 assigned to be sampled on a given number of days post-bolting, and then destructively sampled at 445 that timepoint. Timepoints were spaced every three days, and 3-12 plants sampled for each 446 timepoint. Error bars for all graphs show standard error of the mean. The data presented in Figures $4474 A-D$ are two-timepoint rolling averages of the raw data presented in Figures S2A-D respectively, 448 in order to show slightly smoothed versions of the data, illustrating the overall trend. (A) Scatter 449 graph showing mean opened flowers, at each timepoint from 0-33/36dpb for each genotype. (B) 450 Scatter graph showing the number of total floral nodes present at each timepoint from 0$45124 / 27 / 36 \mathrm{dpb}$ for each genotype. (C) Scatter graph showing the number of unopened primordia 452 present in the inflorescence apex at each timepoint from 0-30/36dpb for each genotype.

453 (E) Box plots showing the total number of opened flowers on the primary inflorescence of Col-0, 454 ahk2 ahk3, rock2, rock3, either untreated (yellow boxes), or treated with inflorescence removal at $4559 \mathrm{dpb}$ (orange boxes), or fruit removal at 14dpb (beige boxes). Boxes indicates the interquartile 456 range, internal line shows the median. Whiskers indicate maximum and minimum values. Bars with 457 the same letter are not statistically different from each other, (ANOVA + Tukey HSD, calculated 458 separately within each genotype, $n=2-9)$.

459 (F) Box plots showing the inflorescence lifetime in days of the primary inflorescence of Col-0, ahk2 460 ahk3, rock2, rock3, either untreated (yellow boxes), or treated with inflorescence removal at 9dpb 461 (orange boxes), or fruit removal at $14 \mathrm{dpb}$ (beige boxes). Boxes indicates the interquartile range, 462 internal line shows the median. Whiskers indicate maximum and minimum values. Bars with the 463 same letter are not statistically different from each other, (ANOVA + Tukey HSD, calculated 464 separately within each genotype, $n=2-9$ ). 

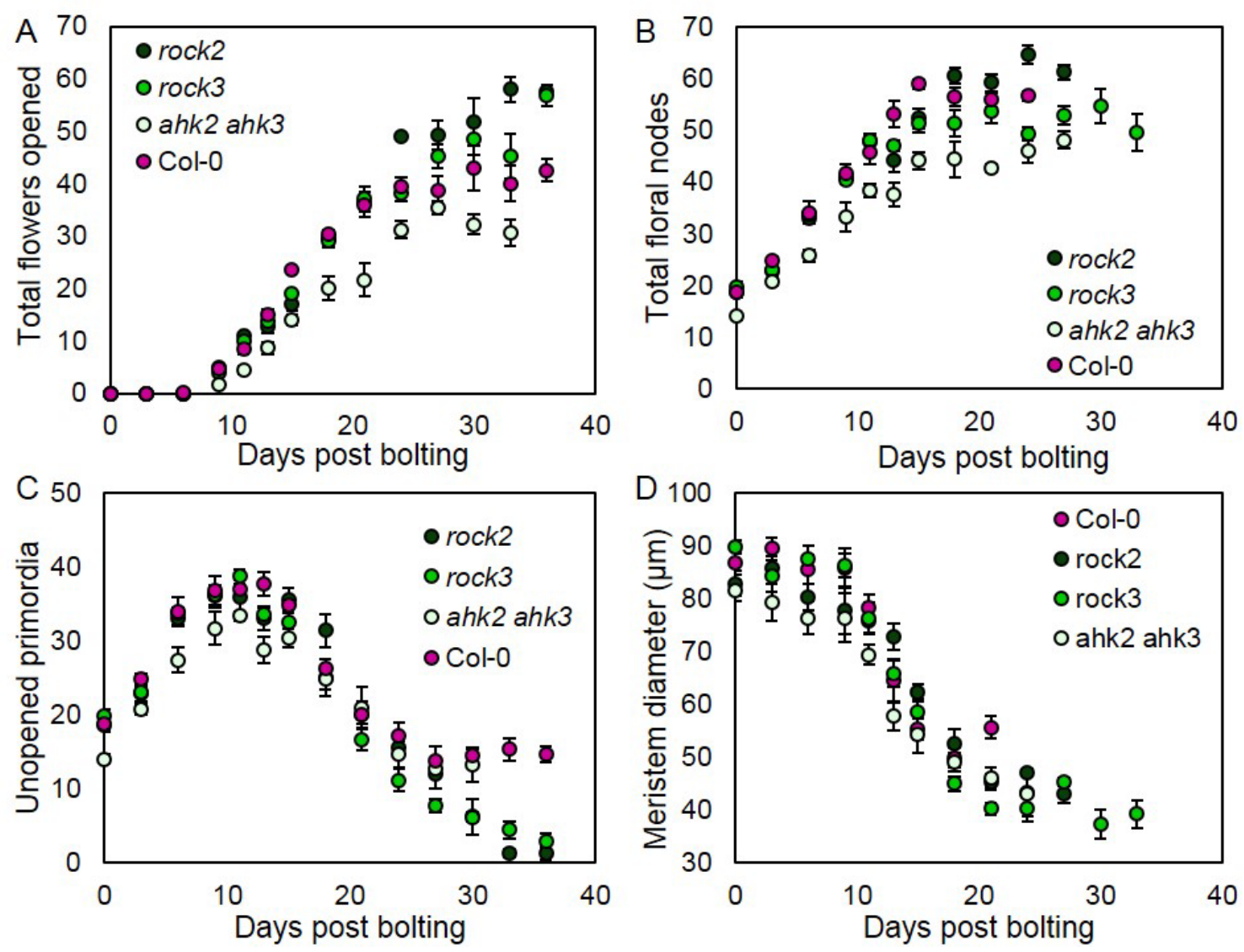

\section{Figure S3: Cytokinin signalling regulates floral arrest}

466 (A-D) Large populations of Col-0, ahk2 ahk3, rock2 and rock3 plants were grown under controlled 467 conditions. The timing of visible bolting was recorded for each plant. Plants were randomly 468 assigned to be sampled on a given number of days post-bolting, and then destructively sampled at 469 that timepoint. Timepoints were spaced every three days, and 3-12 plants sampled for each 470 timepoint. Error bars for all graphs show standard error of the mean. These are the raw data, which 471 are re-drawn in Figures 4A-D as two-timepoint rolling averages of this data. (A) Scatter graph 472 showing mean opened flowers, at each timepoint from 0-33/36dpb for each genotype. (B) Scatter 473 graph showing the number of total floral nodes present at each timepoint from $0-24 / 27 / 36 \mathrm{dpb}$ for 474 each genotype. (C) Scatter graph showing the number of unopened primordia (floral buds and 475 primordia) present in the inflorescence apex at each timepoint from 0-30/36dpb for each genotype. 


\section{DISCUSSION}

477

\section{Two stage inflorescence arrest in Arabidopsis}

479 Previous work has tended to view inflorescence arrest in Arabidopsis as a process driven by changes in the activity of the inflorescence meristem (IMs) [19,20,21]. However, the fact that Arabidopsis inflorescences arrest with a cluster of unopen flowers calls into question this idea. If IM arrest directly led to inflorescence arrest, then inflorescence arrest should occur because a lack of new flowers to open (as is indeed the case in many species). The results presented here clearly demonstrate that inflorescence arrest in Arabidopsis involves the arrest of both IMs and FMs, and show that that the timing of inflorescence arrest is directly determined by the timing of FMl arrest, rather than IM arrest. Our results clearly demonstrate that IM and FM arrest are separate and separable events; local fruit removal can prevent FM arrest, and result in the opening of almost all flowers in the bud cluster. Conversely, global inflorescence removal can delay both IM and floral arrest, resulting in both the initiation and opening of more flowers.

In our previous work, we showed that auxin export from later-produced fruit is locally required for inflorescence arrest [12]. The results presented here demonstrate that this auxin-related mechanism likely specifically relates to FM arrest and not IM arrest, given that we show here that local fruit removal does not affect IM activity (Figure 2). Furthermore, by the point that late fruit are forming, the IM has already arrested. Thus, the auxin exported from late fruit directly inhibits the development of the FMs in the bud cluster, which we show here enter a quiescent state after arrest, from which they can be can be reactivated (Figure 3A). Since initial FM arrest likely occurs shortly after IM arrest (Figure 1), it is likely the case that auxin from late fruit is required to maintain FM arrest, rather than being the stimulus that initiates it. The work presented here thus clarifies the interpretation of our previous work.

\section{Coordination of inflorescence lifetime by systemic cytokinin abundance}

503 Our results also clearly demonstrate that cytokinin is an important regulator of two-stage 504 inflorescence arrest in Arabidopsis. Our results show that cytokinin treatment can delay IM and FM arrest, and that cytokinin mutants show strong perturbations in the progression of inflorescence lifetime (Figure 4, Figure 5). In particular, we show that the rock2 and rock3 mutants, previously implicated in inflorescence arrest [29], differentially regulate IM and FM arrest, consistent with the expression of AHK2 in IMs and FMs, and of AHK3 in FMs. Remarkably, our results show that rock 2 and rock3 phenotypes closely resemble the effect of global inflorescence and local fruit removal respectively, implicating cytokinin in the coordination of arrest events across the plant in response to systemic and local reproductive success. Consistent with this, we observed a clear decline in cytokinin signalling in the IM in the lead-up to IM arrest, and an inability of ahk2 ahk3 mutants to respond to inflorescence or fruit removal. 
515 Overall, our results suggest that cytokinin likely acts as an external factor that causes the arrest of 516 IMs and FMs, though there might be others. Our data suggest a model in which inflorescences and 517 fertile fruit act as sinks that compete for a limited pool of root-derived trans-Zeatin ( $t Z)$. As flowering 518 progresses, and more cytokinin sinks are produced, there is a decline in $t Z$ availability for each 519 organ (Figure 6). Once a critical $t Z$ threshold is reached, IM and FMs arrest is triggered, although 520 flowers that are sufficiently well-developed ( $>6$ days post initiation) can continue to open until 521 visible inflorescence arrest (Figure 6). Our results suggest that distribution of cytokinin within the 522 reproductive shoot system acts as a simple but elegant system that fulfils all three requirements for 523 achieving reproductive success; it coordinates the overall quantity of structures produced, 524 modulates the timing of the reproductive phase, and mediates feedback from reproductive success 525 across the plant. This allows Arabidopsis to flexibly modify inflorescence lifetime in response to 526 local and systemic information, and thereby maximise reproductive success. 


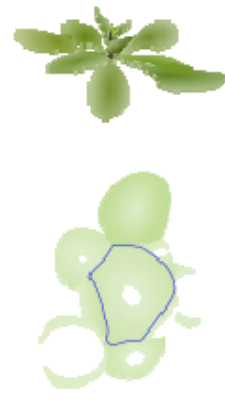

Bolting
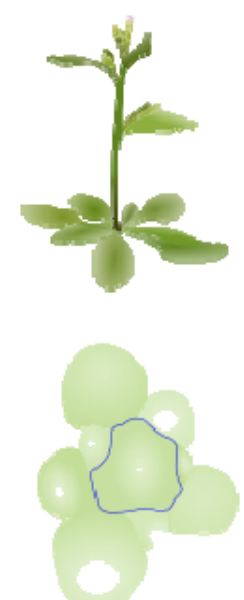

Anthesis
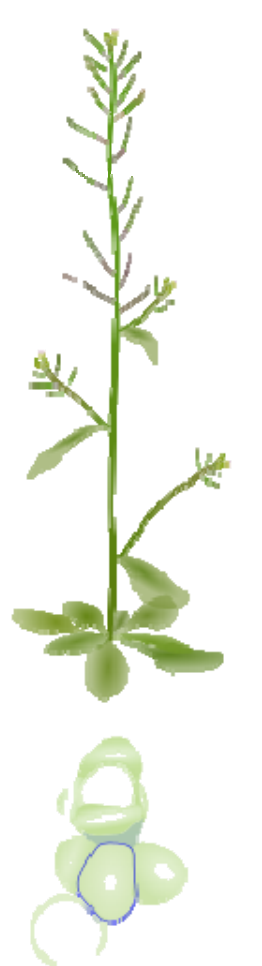

IM arrest

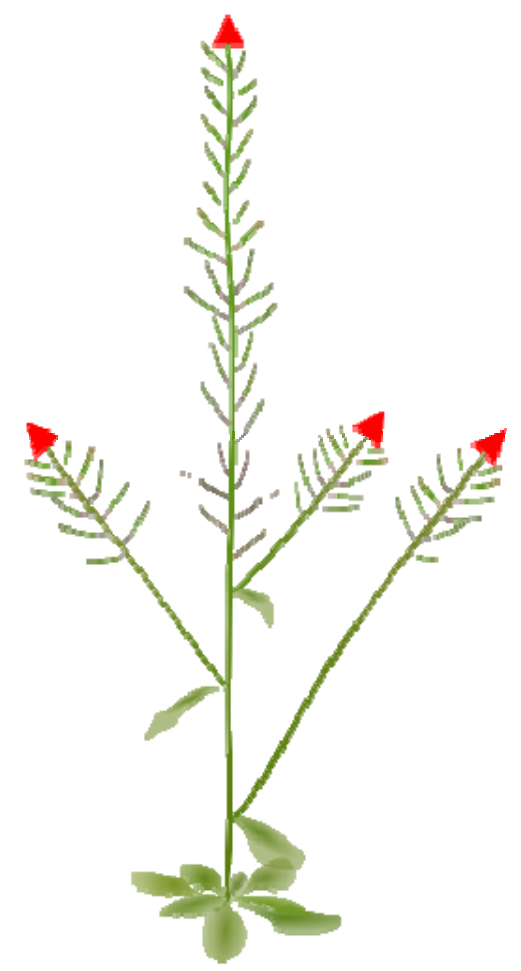

Inflorescence arrest

\section{tZ CK availability/organ}

527 Figure 6: A model for the role of cytokinin in determining inflorescence 528 lifetime

529 Our results suggest a model in which, during early flowering, root-derived trans-Zeatin ( $t Z$ ) 530 cytokinin levels are high, maintaining the size and activity of the primary IM [15]. However, as the 531 size of the shoot system begins to increase, with activation of new inflorescences from 4 days post 532 bolting (dpb) [12], and the development of fruit after anthesis ( 6-7dpb), there are more sinks for $533 t Z$, and less availability in the primary IM. This results in the gradual decrease in size of the IM after

$534 \sim 7 \mathrm{dpb}$, consistent with a reduction in cytokinin signalling in the IM, and ultimately leads to the 535 arrest of the IM ( 12-18 dpb). Shortly afterwards, FMs are also affected by the reduction in $t Z$ 536 availability, triggering arrest in all FMs less than 7 days old; older FMs continue their development, 537 leading to a visible inflorescence arrest ( 17-25 dpb). However, if competing $t Z$ sinks such as 
538 inflorescences are removed early in flowering, sufficient $t Z$ is now available in the primary

539 inflorescence to prolong the activity of the $\mathrm{IM}$, and also to promote the development of almost all

540 FMs. If $t Z$ sinks such as fruit are removed later and more locally, sufficient $t Z$ is liberated to

541 promote the ongoing development of FMs, especially since the inhibitory effect of auxin export

542 from fertile fruit is also removed, but not the IM itself. The hypothesised re-distribution of $t Z$

543 cytokinin between sinks in the shoot would present an elegantly simple system for plants to adjust

544 inflorescence lifetime to compensate for reduced reproductive success. In particular, it can be seen

545 that a local failure of external pollination - not a factor in highly self-fertile Arabidopsis, but a key

546 consideration in most other Brassicaceae - would trigger the compensatory maturation of

547 additional flowers by preventing cytokinin sinks/auxin sources developing. A more dramatic loss of

548 inflorescences by e.g. herbivory would trigger both the development of additional inflorescences [2]

549 and prolong the lifetime of existing inflorescences. 


\section{MATERIALS \& METHODS}

551

\section{Plant growth conditions}

553 Plants for phenotypic and microsurgical experiments were grown on John Innes compost under a 554 standard $16 \mathrm{~h} / 8 \mathrm{~h}$ light/dark cycle $\left(20^{\circ} \mathrm{C}\right)$ in either controlled environment rooms with light provided 555 by fluorescent tubes at a light intensity of $\sim 120 \mu \mathrm{mol} / \mathrm{m}^{2} \mathrm{~s}^{-1}$, or in glasshouses with supplemental 556 lighting. Plants for cytokinin application experiments were grown on John Innes No. 3 compost 557 under the same light/dark cycle but at $22^{\circ} \mathrm{C} / 18^{\circ} \mathrm{C}$, with light provided by fluorescent tubes at an 558 intensity of $\sim 150 \mu \mathrm{mol} / \mathrm{m}^{2} \mathrm{~s}^{-1}$.

559

\section{Plant materials}

561 Arabidopsis wild-types Col-0 and Ler were used as indicated. The following lines are all in a Col-0 562 background and have previously been described; TCSn:GFP [28]; arr1-4 [31]; rock2 [29]; rock3 563 [29]; ipt3-2 ipt5-2 ipt7-1 [36]; ahk2-2 ahk3-3 [32].

\section{Flowering assessments and meristem measurements}

566 To define the manner in which Arabidopsis inflorescences arrest, we grew a large population of 567 wild-type Col-0 Arabidopsis under long day conditions. Each plant was pre-allocated to be sampled 568 at a given timepoint after its primary shoot axis had 'bolted'). In this way 6 plants were sampled 569 for each timepoint, with the timepoints being at 1 day intervals post bolting. Sampling was 570 destructive, so we could not just measure the same plants each day post bolting (dpb). For each 571 plant we recorded 1) the number of open and previously opened flowers; 2) the number of as-yet572 unopened floral buds including all floral primordia visible by dissecting the inflorescence apex 573 under a microscope (Figure 1F); and 3) the cumulative number of floral nodes initiated by each 574 inflorescence at that timepoint (i.e. the sum of 1 and 2).

575

576 Genotypes (where relevant) and age of collection were randomised across trays, and date of 577 bolting recorded for each plant. When ready for collection, the entire bud cluster above the 578 uppermost open flower (where present) was removed from the plant with forceps. In the event of 579 collection prior to flowering, the entire bud cluster was collected. All open flowers on the primary 580 inflorescence (PI) were counted prior to collection. The apex of the inflorescence (containing all 581 unopened flowers) was removed from each plant and mounted into a plate containing solidified 582 water agar to prevent dessication, with the meristem facing upwards. These were then dissected 583 under a dissecting microscope using forceps and micro-scalpel. The total number of unopened 584 flowers and floral primordia were counted, with as many as possible being removed. The dissected 585 apices were imaged under a Keyence VHX-7000 digital microscope, using a VH-Z100R RZx100- 
586

587

588

589

590

591

592

593

594

595

596

\section{7}

598

599

600

601

602

603

604

605

606

607

608

609

610

611

612

613

614

615

616

617

618

619

620

x1000 real zoom lens. Images were loaded into ImageJ [37], where the mean of three meristem diameters was calculated, using methodology adapted from Landrein et al [38].

\section{Micro-surgical experiments}

Inflorescence removal as described in Figures 2 and 4 was carried out by removing all inflorescences except the PI with scissors at 6 DPB. Plants were then monitored every subsequent 2-3 days and newly developed branches were removed until sample collection. Fruit removal treatments were carried out at either 14 DPB or on the day of final flower opening as indicated. All developed fruits and open flowers were removed from the PI using forceps. Plants were monitored every 1-3 days, with all additional flowers being removed until sample collection.

\section{Confocal imaging}

Inflorescence apices of TCSn:GFP plants were prepared, mounted and dissected as described above. The agar plates were then flooded with distilled water to allow water-dipping lenses to be used to image the meristem. Meristems were imaged using a Zeiss LSM880 with a 20x water dipping lens. Excitation was performed using $488 \mathrm{~nm}$ (10\% laser power) and $555 \mathrm{~nm}(5 \%)$ lasers. Chloroplast autofluorescence was detected above $600 \mathrm{~nm}$, and GFP fluorescence below $555 \mathrm{~nm}$. Z-stacks were taken of each meristem, covering the whole depth of the meristem dome, and then a maximum intensity projection was made of the z-stack. Quantification was performed on these projections using imageJ. The microscope same settings were used for all meristems.

\section{qPCR}

Col-0 plants were grown and their date of anthesis recorded. Inflorescence apices (including all unopened buds) of the PI (4-8 individual plants pooled per biological replicate) were subsequently harvested, snap frozen, and stored at -80 degrees Celsius until RNA extraction. RNA was extracted from samples using a QIAGEN RNeasy plant mini kit as per manufacturer's instructions (including DNAse treatment). cDNA was synthesised using Superscript IV reverse transcriptase with 1microgram of input RNA per sample. qRT-PCR was performed on an Analytik-Jena qTOWER using PowerUp SYBR Green mastermix (Thermo-Fisher), with 10ul reactions containing

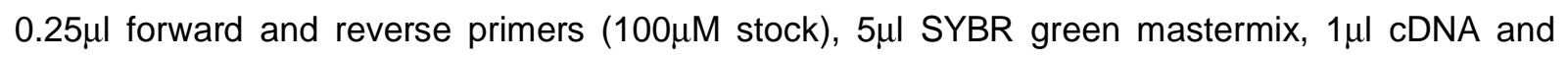
$3.5 \mu \mathrm{l}$ water. $\mathrm{Cp}$ values were calculated using the manufacturer's software and subsequently compared via the $2^{-\Lambda \Lambda C t}$ method, normalised to the average day 0 values, with the housekeeping gene PP2A3 as an internal control. Results presented are the average of four biological replicates with three technical replicates each. 
621 Primers: ARR5-F - tcagagaacatcttgcctcgt; ARR5-R - atttcacaggcttcaataagaaat; ARR7-F -

622 ccggtggagatttgactgtt; ARR7-R - tccactctctacagtcgtcacttt; PP2A3-F - tccgtgaagctgctgcaaac;

623 PP2A3-R - caccaagcatggccgtatca.

624

\section{Cytokinin applications}

626 Cytokinin applications were performed via application in lanolin to emerged fruits of Ler plants 627 using a micropipette tip, the same methodology as in Ware et al [12]. Either $10 \mathrm{ul}(1 \mathrm{mg} / \mathrm{g}$ treatment) 628 or $1 \mathrm{ul}(01 \mathrm{mg} / \mathrm{g}$ treatment) of $100 \mathrm{mg} / \mathrm{ml} 6$-benzlyaminopurine stock in DMSO was added to lanolin 629 with 1 ul dye to ensure even incorporation, or DMSO and dye alone for the corresponding mock 630 treatments. Treatments were performed at the same points as measurements, and the treatment 631 regimen was initiated at 12 days after anthesis of the first flower.

632

\section{Cytokinin measurements}

634 For the cytokinin analysis of the fertile or sterile fruit of Ler and ms 1 plants approximately $10 \mathrm{mg}$ of 635 fresh weight material was used per sample $(n=5)$. Samples were extracted in modified Bieleski 636 buffer (methanol/water/formic acid, 15/4/1, (v/v/v)) with a mixture of stable isotopically labelled 637 internal standards added to each sample for precise quantification [39]. The purification of 638 isoprenoid CKs was carried out according to [40] using the MCX column (30 mg of C18/SCX 639 combined sorbent with cation-exchange properties). Analytes were eluted by two-step elution using 640 a $0.35 \mathrm{M} \mathrm{NH}_{4} \mathrm{OH}$ aqueous solution and $0.35 \mathrm{M} \mathrm{NH}_{4} \mathrm{OH}$ in $60 \% \mathrm{MeOH}(\mathrm{v} / \mathrm{v})$ solution. Samples were 641 afterwards evaporated to dryness under vacuum at $37^{\circ} \mathrm{C}$. Prior analysis the samples were 642 dissolved in $40 \mu \mathrm{l} 10 \% \mathrm{MeOH}(\mathrm{v} / \mathrm{v})$. MS analysis and quantification were performed using an 643 UHPLC-MS/MS system consisting of a 1290 Infinity Binary LC System coupled to a 6490 Triple 644 Quad LC/MS System with Jet Stream and Dual Ion Funnel technologies (Agilent Technologies, 645 Santa Clara, CA, USA. UHPLC-ESI-MS/MS method parameters were adapted from [41].

\section{Experimental design and statistics}

648 Sample size for each experiment is described in the figure legends. For plant growth experiments, 649 each sample was a distinct plant. For cytokinin measurements, each sample was set of tissue 650 pooled from multiple plants; each sample was distinct. For data analysis, we tested data for 651 normality to determine the most appropriate statistical test, except when mixed-effects models 652 were used, where instead sphericity was not assumed and the Greenhouse-Geisser correction 653 was applied. For Sidak's multiple comparisons, individual variances were calculated for each 654 comparison.

655 
657 All figures in this manuscript are associated with raw data. All data will be made available upon

658 request.

659

660

661

\section{ACKNOWLEDGEMENTS}

$663 \mathrm{CHW}$ is supported by BBSRC White Rose PhD studentship (BB/M011151/1). AW is supported by

664 BBSRC DTP grant BB/M008770/1. JS and KL are supported by the Knut and Alice Wallenberg

665 Foundation (KAW), the Swedish Foundation for Strategic Research (Vinnova), and the Swedish

666 research council (VR). We also acknowledge the Swedish Metabolomics Centre

667 (http://www.swedishmetabolomicscentre.se/) for access to instrumentation.

668

669 AUTHOR CONTRIBUTIONS

$670 \mathrm{CHW}, \mathrm{AW}, \mathrm{TB}$ performed experiments and analysed the data. JS, KL carried out the cytokinin

671 analysis. TB \& ZW designed the study. All authors contributed to writing the manuscript.

672

673 COMPETING INTERESTS

674 The authors declare that they have no competing interests. 


\section{REFERENCES}

1. Walker, C.H. and Bennett, T. (2018). Forbidden fruit: dominance relationships and the control of shoot architecture. Annual Plant Reviews Online, doi: 10.1002/9781119312994.apr0640.

2. Walker, C.H., Wheeldon, C.D. and Bennett, T. (2021). Integrated dominance mechanisms regulate reproductive architecture in Arabidopsis thaliana and Brassica napus. Plant Physiology, 186, 1985-2002.

3. Takei, K., Sakakibara, H., Taniguchi, M. and Sugiyama, T. (2001). Nitrogen-dependent accumulation of cytokinins in root and the translocation to leaf: implication of cytokinin species that induces gene expression of maize response regulator. Plant and Cell Physiology, 42, 85-93.

4. Umehara, M., Hanada, A., Magome, H., Takeda-Kamiya, N. and Yamaguchi, S. (2010). Contribution of strigolactones to the inhibition of tiller bud outgrowth under phosphate deficiency in rice. Plant and Cell Physiology, 51, 1118-1126.

5. Wheeldon, C.D. and Bennett, T. (2021). There and back again: an evolutionary perspective on long-distance coordination of plant growth and development. Seminars in Cell \& Developmental Biology, 109, 55-67.

6. Prusinkiewicz P, Crawford S, Smith RS, Ljung K, Bennett T, Ongaro V, Leyser O (2009). Control of bud activation by an auxin transport switch. PNAS, 106, 17431-17436.

7. Shinohara N, Taylor C, Leyser O (2013). Strigolactone can promote or inhibit shoot branching by triggering rapid depletion of the auxin efflux protein PIN1 from the plasma membrane. PLoS Biology 11, e1001474.

8. Bennett T, Hines G, van Rongen M, Waldie T, Sawchuk MG, Scarpella E, Ljung K, Leyser O. (2016). Connective auxin transport in the shoot facilitates communication between shoot apices. PLoS Biol, 14, e1002446.

9. Van Rongen, M., Bennett, T., Ticchiarelli, F. and Leyser, O. (2019). Connective auxin transport contributes to strigolactone-mediated shoot branching control independent of the transcription factor BRC1. PLoS Genetics, 15, e1008023.

10. Bangerth, F (1989). Dominance amongst fruits/sinks and the search for a correlative signal. Physiologia Plantarum, 76, 608-614. 
11. Lenser T, Tarkowská D, Novák O, Wilhelmsson PKI, Bennett T, Rensing SA, Strnad M, Theißen G. (2018). When the BRANCHED network bears fruit: how carpic dominance causes fruit dimorphism in Aethionema. Plant Journal, 94, 352-371.

12. Ware, A., Walker, C.H., Šimura, J., González-Suárez, P., Ljung, K., Bishopp, A., Wilson, Z.A. and Bennett, T. (2020). Auxin export from proximal fruits drives arrest in temporally competent inflorescences. Nature Plants, 6, 699-707.

13. Haim, D., Shalom, L., Simhon, Y., Shlizerman, L., Kamara, I., Morozov, M. et al. (2021). Alternate bearing in fruit trees: fruit presence induces polar auxin transport in citrus and olive stem and represses IAA release from the bud. Journal of Experimental Botany, 72, 2450-2462.

14. Goetz, M., Rabinovich, M. and Smith, H.M. (2021). The role of auxin and sugar signaling in dominance inhibition of inflorescence growth by fruit load. Plant Physiology, 187, 1189-1201.

15. Landrein, B., Formosa-Jordan, P., Malivert, A., Schuster, C., Melnyk, C.W., Yang, W. et al. (2018). Nitrate modulates stem cell dynamics in Arabidopsis shoot meristems through cytokinins. PNAS, 115, 1382-1387.

16. Cho, L-H., Yoon, J. and An, G. (2016). The control of flowering time by environmental factors. The Plant Journal, 90, 708-719.

17. Gol, L., Tomé, F. and von Korff, M. (2017). Floral transitions in wheat and barley: interactions between photoperiod, abiotic stresses, and nutrient status. Journal of Experimental Botany, 68, 1399-1410.

18. González-Suárez, P., Walker, C.H. and Bennett, T. (2020). Bloom and bust: understanding the nature and regulation of the end of flowering. Current Opinion in Plant Biology, 57, 24-30.

19. Hensel LL, Nelson MA, Richmond TA, Bleecker AB (1994). The fate of inflorescence meristems is controlled by developing fruits in Arabidopsis. Plant Physiology, 106, 863-76.

20. Wuest, S.E., Philipp, M.A., Guthörl, D., Schmid, B., Grossniklaus, U. (2016) Seed production affects maternal growth and senescence in Arabidopsis. Plant Physiology, 171, 392-404.

21. Balanzà, V., Martínez-Fernández, I., Sato, S., Yanofskym M.F., Kaufmann, K., Angenent, G.C., Bemer, M. and Ferrándiz, C. (2018) Genetic control of meristem arrest and life span in Arabidopsis 
by a FRUITFULL-APETALA2 pathway. Nature Communications, 9, doi: 10.1038/s41467-01803067-5.

22. Wang, Y., Kumaishi, K., Suzuki, T., Ichihashi, Y., Yamaguchi, N., Shirakawa, M. and Ito, T. (2020). Morphological and physiological framework underlying plant longevity in Arabidopsis thaliana. Frontiers in Plant Science 11, 600726

23. Smyth, D.R., Bowman, J.L., Meyerowitz, E.M. (1990). Early flower development in Arabidopsis. Plant Cell 2, 755-767

24. Mandel, T., Moreau, F., Kutsher, Y., Fletcher, J.C., Carles, C.C. and Williams, L.E. (2014). The ERECTA response kinase regulates Arabidopsis shoot apical meristem size, phyllotaxy and floral meristem identity. Development, 141, 830-841.

25. Mandel, T., Candela, H., Landau, U., Asis, L., Zelinger, E., Carles, C.C. and Williams, L.E. (2016). Differential regulation of meristem size, morphology and organization by the ERECTA, CLAVATA and class III HD-ZIP pathways. Development, 143, 1612-1622.

26. Zhang, L., DeGennaro, D., Lin, G., Chai, J. and Shpak, E.D. (2021). ERECTA family signaling constrains CLAVATA3 and WUSCHEL to the center of the shoot apical meristem. Development, 148, doi: 10.1242/dev.189753.

27. Müller, D., Waldie, T., Miyawaki, K., To, J.P.C., Melnyk, C.W., Kieber, J.J., Kakimoto, T. and Leyser, O. (2015). Cytokinin is required for escape but not release from auxin mediated apical dominance. The Plant Journal, 82, 874-886.

28. Liu, J. and Müller, B. (2017). Imaging TCSn::GFP, a synthetic cytokinin reporter, in Arabidopsis thaliana. Methods in Molecular Biology, 1497, 81-90.

29. Bartrina, I., Jensen, H., Novák, O., Strnad, M., Werner, T. and Schmülling, T. (2017). Gain-offunction mutants of the cytokinin receptors AHK2 and AHK3 regulate plant organ size, flowering time and plant longevity. Plant Physiology, 173, 1783-1797.

30. Waldie T, Leyser O. (2018). Cytokinin Targets Auxin Transport to Promote Shoot Branching. Plant Physiology, 177, 803-818. 
31. Hirose N, Takei K, Kuroha T, Kamada-Nobusada T, Hayashi H, Sakakibara H. (2008). Regulation of cytokinin biosynthesis, compartmentalization and translocation, Journal of Experimental Botany 59, 75-83,

32. Higuchi M, Pischke MS, Mähönen AP, Miyawaki K, Hashimoto Y, Seki M. et al. (2004). In planta functions of the Arabidopsis cytokinin receptor family. PNAS, 101, 8821-8826.

33. Nishimura, C., Ohashi, Y., Sato, S., Kato, T., Tabata, S. and Ueguchi, C. (2004). Histidine kinase homologs that act as cytokinin receptors possess overlapping functions in the regulation of shoot and root growth in Arabidopsis. The Plant Cell, 16, 1365-1377.

34. Schmid M, Davison TS, Henz SR, Pape UJ, Demar M, Vingron M, Schölkopf B, Weigel D, Lohmann JU. (2005). A gene expression map of Arabidopsis thaliana development. Nature Genetics 37, 501-506.

35. Zhang, W., To, J.P., Cheng, C.Y., Schaller, G.E. and Kieber, J.J. (2011). Type-A response regulators are required for proper root apical meristem function through post-transcriptional regulation of PIN auxin efflux carriers. Plant Journal, 68, 1-10.

36. Miyawaki, K., Tarkowski, P., Matsumoto-Kitano, M., Kato, T., Sato, S., Tarkowska, D., Tabata, S., Sandberg, G. and Kakimoto, T. (2006). Roles of Arabidopsis ATP/ADP isopentenyltransferases and tRNA isopentenyltransferases in cytokinin biosynthesis. PNAS, 103, 16598-16603.

37. Schneider, C. A., Rasband, W. S., \& Eliceiri, K. W. (2012). NIH Image to ImageJ: 25 years of image analysis. Nature Methods, 9, 671-675.

38. Landrein, B., Refahi, Y., Besnard, F., Hervieux, N., Mirabet, V., Boudaoud, A., Vernoux, T., Hamant, O. (2015). Meristem size contributes to the robustness of phyllotaxis in Arabidopsis.

Journal of Experimental Botany 66, 1317-1324.

39. Hoyerová, K., Gaudinová, A., Malbeck, J., Dobrev, P.I., Kocábek, T., Šolcová, B., Trávníčková, A., Kamínek, M. (2006). Efficiency of different methods of extraction and purification of cytokinins. Phytochemistry, 67, 1151-1159.

40. Dobrev, P.I., Kamínek, M. (2002). Fast and efficient separation of cytokinins from auxin and abscisic acid and their purification using mixed-mode solid-phase extraction. Journal of Chromatography A, 950, 21-29. 

available under aCC-BY-NC-ND 4.0 International license.

41. Svačinová, J., Novák, O., Plačková, L., Lenobel, R., Holík, J., Strnad, M., \& Doležal, K. (2012). A new approach for cytokinin isolation from Arabidopsis tissues using miniaturized purification: pipette tip solid-phase extraction. Plant Methods, 8, 17. 